Journal of Behavioral Decision Making, Vol. 8, 201-216, (1995)

\title{
Dominance Violations in Judged Prices of Two- and Three-outcome Gambles
}

\author{
BARBARA A. MELLERS \\ University of California at Berkeley, USA \\ PATRICIA M. BERRETTY \\ University of California, Santa Barbara, USA \\ MICHAEL H. BIRNBAUM \\ California State University, Fullerton, USA
}

\begin{abstract}
The dominance principle states that one should prefer the option with consequences that are at least as good as those of other options for any state of the world. When applied to judged prices of gambles, the dominance principle requires that increasing one or more outcomes of a gamble should increase the judged price of the gamble, with everything else held constant. Previous research has uncovered systematic violations of the dominance principle: people assign higher prices to a gamble with a large probability of winning an amount, $Y$, otherwise zero, than they do to a superior gamble with the same chance of winning $Y$, otherwise winning a small amount, $X$ ! These violations can be explained by a configural-weight theory in which two-outcome gambles are represented with two sets of decision weights; one set for outcomes having values of zero and another set for lower-valued outcomes that have nonzero values. The present paper investigates whether dominance violations are limited to two-outcome gambles. Results show that people violate the dominance principle with three-outcome gambles even with financial incentives. Furthermore, results could be predicted from the configural-weight theory. The data do not support the view that configural weighting is caused by a shift in strategy that would apply only to two-outcome gambles.
\end{abstract}

KEY WORDS dominance principle; two-outcome gambles; three-outcome gambles; configural-weight theory

\section{INTRODUCTION}

The dominance principle (also called monotonicity) is one of the most fundamental principles of decision making. It states that decision makers should choose the option for which the consequences are as good or better than those of other options, for any state of the world. The value of an option should strictly increase as one of its consequences improves, with all else held constant. Although much past research has shown that people violate normative rules of decision making (Arkes and Hammond, 1986; 
Bell et al., 1988), few tests of dominance were performed, perhaps because the principle seems like common sense.

Recently, Birnbaum et al. (1992) found that people systematically violated the dominance principle: they assigned higher prices to an inferior gamble with a large probability of winning an amount, $Y$, otherwise zero, than to a superior gamble gambles with the same chance of winning $Y$, otherwise winning a small amount, $X$. For example, subjects paid more money to play a gamble with a $95 \%$ chance of $\$ 96$, otherwise $\$ 0$, than they did to play a better gamble with the same chance of $\$ 96$, otherwise $\$ 24$ ! These violations were obtained in all three points of view studied by Birnbaum et al. (1992): buyer's prices (what is the most that a buyer is willing to pay for the gamble?), seller's price (what is the least that a seller will accept?), and neutral price (what is the 'fair' price?).

These surprising violations are not restricted to the domain of gains. Mellers et al. (1992a) found similar effects with avoidance prices for gambles in the domain of losses (i.e. analogous to insurance premiums). Subjects paid more money to avoid playing the gamble with a $95 \%$ chance of losing $\$ 96$, otherwise zero, than they did to avoid playing the gamble with the same chance of losing $\$ 96$, otherwise losing $\$ 24$. In both gains and losses, violations of dominance persisted, despite different stakes, different information displays, and different subject populations. Even with financial incentives, people systematically violated this basic rule of decision making. However, they did not attempt to defend their judgments in post-experimental interviews.

In the experiments described above, subjects made a large number of judgments. When subjects make only a few judgments, dominance violations are rare. With only a small set of gambles, one is more likely to compare judgments and detect the dominance relation. Dominance violations are also infrequent with direct choices between pairs of gambles (Birnbaum and Sutton, 1992). Von Winterfeldt et al. (1994) found that violations were less frequent with a certain form of choice-based certainty equivalents based on repeated choices between sure things and gambles. However, Birnbaum (1992) demonstrated that systematic violations could occur with another form of choice-based certainty equivalents in which subjects were presented with a gamble and a fixed set of sure things, with instructions to indicate which of the sure things they would prefer to the gamble. These results were replicated and extended to choice proportions by Birnbaum and Thompson (in press). Thus, when the dominance relation is unclear, dominance violations often occur, and when the relation is transparent, as in direct choices or side-byside judgments, violations are substantially reduced.

\section{Configural-weight theory}

Configural-weight theory is a rival to additive and simple averaging theories with constant weights (Birnbaum, 1973, 1974; Birnbaum and Stegner, 1979). With additive and simple averaging theories (e.g. Savage's, 1954, subjective expected utility theory), the weight of a stimulus, such as an outcome, is assumed to be independent of its value and the stimulus configuration, or the relationship between the outcome and the other outcomes in the gamble. In configural-weight theory, however, the weight of a stimulus can depend on its value and the stimulus configuration. Rank-dependent and rank- and sign-dependent theories of choice are types of configural weighting that were developed independently (Quiggin, 1982; Yaari, 1987; Lopes, 1984; Luce and Narens, 1985; Luce, 1991; Luce and Fishburn, 1991; Wakker, 1994; Tversky and Kahneman, 1992). It should be noted, however, that many of the recent rank-dependent and rank- and sign-dependent theories are founded on the axiom of monotonicity, so they cannot explain violations of dominance without giving up this key premise on which they are based.

Configural-weight theory can be derived from the rationale that subjects act as if they are minimizing an asymmetric loss function (Birnbaum et al., 1992). The configural-weight parameter, $a_{v}$, represents the asymmetry of the loss functions. The cost to buyers of paying too much for a gamble that loses (buyer's remorse) is often greater than the cost of offering too little for the gamble and not getting the opportunity 
to win. In contrast, sellers are often more concerned with selling a gamble for too little when the gamble wins (seller's lament) than with holding out for a higher price. Configural-weight theory accounts for changes in judged values from different points of view (buyers versus sellers); it also explains violations of branch independence (a strong form of independence of common consequence); and it predicts violations of monotonicity.

For two-outcome gambles with $X<Y$, configural-weight theory (Birnbaum et al., 1992) can be written:

$$
\mathrm{U}(X, P, Y)=(A u(X)+B u(Y)) /(A+B)
$$

where $\mathrm{U}(X, P, Y)$ is the utility of a gamble with probability, $P$, to win $X$, otherwise $Y$ with probability, $Q$, $A$ and $B$ are the configural weights of the lower and higher outcomes, respectively. They can be expressed:

and

$$
A=a_{\mathrm{v}} S_{X}(P)
$$

$$
B=\left(1-a_{\mathrm{v}}\right)\left(1-S_{X}(1-Q)\right)
$$

where $a_{\mathrm{v}}$ is the configural-weight parameter that represents the judge's point of view, $V ; S_{X}(P)$ is the weighting function for the lowest outcome in the configuration of outcomes, which depends on probability, $P$.

Birnbaum et al. used two $S_{X}(P)$ functions, one for the case in which $X=0$, and the other for $X>0$. Estimated weights for zero-valued outcomes $(X=0)$ were smaller than the corresponding weights for nonzero outcomes. Under certain conditions, people appear to place less weight on zero-valued outcomes relative to nonzero outcomes, especially when the probabilities of their occurrence are small.

For three-outcome gambles, Birnbaum et al. (1992) used the following extension:

$$
\mathrm{U}(X, P ; Y, Q ; Z)=(A u(X)+B u(Y)+C u(Z) /(A+B+C)
$$

where $\mathrm{U}(X, P ; Y, Q ; Z)$ is the utility of the gamble to receive $X$ (the lowest outcome) with probability, $P$; $Y$ with probability $Q$, and $Z$ with probability $1-P-Q ; A$ and $B$ are the same as above, and $C$, the weight of outcome $Z$, is:

$$
C=\left(1-a_{\mathrm{v}}\right)\left(1-S_{X}(1-(1-P-Q))\right)
$$

In the terminology of configural-weight theory, $A, B$, and $C$ are absolute configural weights, and relative configural weights are defined as each weight divided by the sum of the absolute weights (e.g. $A /(A+B)$ or $A /(A+B+C))$.

\section{Do dominance violations occur with three-outcome gambles?}

Although Birnbaum et al. (1992) formulated configural-weight theory for three outcomes and showed how it could account for violations of branch independence in three-outcome gambles, they did not examine whether violations of dominance occurred in three-outcome gambles. The theory implies that dominance violations should persist with three-outcome gambles, but a rival intuition suggests that they might not occur. When people evaluate two-outcome gambles having one zero outcome, they may use a simple strategy, multiplying probability by amount. However, if there are two or more nonzero outcomes, people may initially calculate an average of the outcomes, and then adjust the average to estimate the value of the gamble.

This interpretation of configural-weight theory implies that the weighing functions for $X=0$ is only applied to two-outcome gambles. Since three-outcome gambles with one zero outcome do not invite the 
simple multiplicative strategy, subjects are presumed to use an averaging strategy for any three-outcome gamble with the same decision weights. That is, the addition of a third outcome makes the gamble sufficiently complex that an outcome of zero is no longer treated differently.

In the present study, we test for dominance violations in three-outcome gambles. Predictions of configural-weight theory for gambles in our experiment are based on approximate parameter values from Birnbaum et al. (1992). This tactic is conservative, since any deficiency in the estimated parameters from previous research or any difference in the value of $a_{\mathrm{v}}$ between the two studies would cause the predictions to deviate from the data for technical rather than theoretical reasons.

Birnbaum et al. (1992) assumed that $u(X)=X$ for all three points of view, and that judgments of value are a linear function of the utilities of the gambles. To extrapolate from the values of $P$ in Birnbaum $e t$ $a l$, we will use a simple linear approximation of the derived weights for the seller's point of view. The linear approximations for the weight of the lowest outcome for values of $0.04<P<0.96$ are:

$$
\begin{aligned}
& S_{X}(P)=0.59 P+0.29 \text { for } X>0 \\
& S_{0}(\mathrm{P})=0.74 P+0.14 \text { for } \mathrm{X}=0
\end{aligned}
$$

These linear functions are shown in Exhibit 1; values represent the relative weight of the lower outcome in two-outcome gambles from the seller's point of view. (For the seller's point of view, $a_{\mathrm{v}}$ was set to 0.5 ; for the buyer's and neutral's point of view, $a_{\mathrm{v}}$ was estimated to be 0.7 and 0.6 , respectively.) Exhibit 1 shows that when the lowest outcome in a gamble is zero, it receives less weight than when it is nonzero. When these parameters are applied to three-outcome gambles, violations of monotonicity are predicted to occur.

We will now illustrate the predictions of configural-weight theory for two- and three-outcome gambles. Consider the gambles $(\$ 24,0.05 ; \$ 96)$ and $(\$ 0,0.05 ; \$ 96)$ which represent a 0.95 probability to win $\$ 96$ and a 0.05 probability to win $\$ 24$ in the first case, and $\$ 0$ in the second. According to equation (1), using $u(X)=X$ and the weights in Exhibit 1, the predicted value of the gamble with $X=\$ 24$ is $(0.5$ $\left.S_{X}(0.05) 24+0.5\left(1-S_{X}(1-0.95)\right) 96\right) /\left(0.5 S_{X}(0.05)+0.5\left(1-S_{X}(1-0.95)\right)\right)$. Since $S_{X}(0.05)=0.32$ and $1-S_{X}(0.05)=0.68$, the predicted value of $(\$ 24,0.05 ; \$ 96)$ is 73 .

Now consider the inferior gamble in which $\$ 24$ has been replaced by $\$ 0$. The value is $\left(0.5 S_{0}(0.05) 0+\right.$ $\left.\left(1-S_{0}(1-0.95)\right) 96\right) /\left(0.5 S_{0}(0.05)+\left(1-S_{0}(1-0.95)\right)\right)$. We know that $S_{0}(0.05)=0.18$ and $1-S_{0}(0.05)$

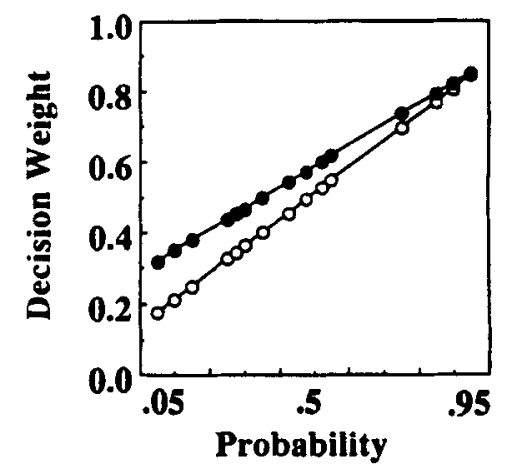

Exhibit 1. Decision weights for zero-valued outcomes (open circles) and nonzero outcomes (filled circles), plotted as a function of probability, when those outcomes are the worst possible outcomes of the gamble. Curves show a linear approximation of empirical weights estimated from Birnbaum et al. (1992, Figure 11) for the seller's point of view. These (approximate) values from previous research will be extrapolated to predict the results of the new experiments with three outcomes 
$=0.82$, so the predicted value of $(\$ 0,0.05 ; \$ 96)$ is $\$ 79$, a higher value than that predicted for the superior gamble.

In a similar way, configural-weight theory predicts violations of dominance with three-outcome gambles. Consider the gambles $(\$ 24,0.05 ; \$ 90,0.475 ; \$ 96)$ and $(\$ 0,0.05 ; \$ 90,0.475 ; \$ 96)$. The predicted value for the first gamble is $(A u(24)+B u(90)+C u(96)) /(A+B+C)$. Since $1-S_{X}(0.525)=0.40$, the value of this gamble is $\left.\left(0.32^{*} 24+0.40^{*} 90+0.40^{*} 96\right)\right) /(0.32+0.40+0.40)=73$. For the dominated gamble, the prediction is $(A u(0)+B u(90)+C u(96)) /(A+B+C)$, which because $1-S_{0}(0.525)=0.47$, can be expressed as $\left.\left(0.18^{*} 0+0.47^{*} 90+0.47^{*} 96\right)\right) /(0.18+0.47+0.47)=78$. Therefore, the predicted value of $\$ 78$ for the inferior gamble exceeds the predicted value of $\$ 73$ for the superior gamble.

\title{
EXPERIMENT 1: TWO-OUTCOME GAMBLES
}

Experiment 1 is designed to replicate previous studies showing dominance violations with two-outcome gambles with both positive and negative expected values.

\begin{abstract}
Method
Instructions

Subjects were told to imagine that they owned a set of gambles, some with positive expected values and other with negative expected values. Instructions stated that they would play each gamble unless they either sold it (in the case of desirable gambles) or paid someone else to play it (in the case of undesirable gambles). The task was to state the value of each gamble from this ownership point of view. For desirable gambles, subjects stated the minimum amounts they would be willing to accept to sell each gamble; for undesirable gambles, subjects stated the maximum amounts they would be willing to play to avoid playing each gamble (analogous to buying insurance). All responses were made in dollars and cents.
\end{abstract}

\section{Stimuli and design}

The probability device was represented as a pie chart with an imaginary spinner, as Figure 1 of Mellers $e t$ al. (1992b). Winning or losing depended on whether the imaginary spinner landed in the black or grey regions of the pie chart. The proportion of the black region was varied to match $P$, the probability of winning (or losing) the largest absolute amount. Amounts to win (or lose) were indicated beside each gamble.

Gambles were constructed from six factorial designs, as in Experiment 4 of Mellers et al. (1992a). The first was a $5 \times 9$, Probability by Amount-Pairs design. Probabilities of winning the larger amount were $0.05,0.20,0.50,0.80$, and 0.95. Amount pairs were $(\$ 96, \$ 0),(\$ 96, \$ 6),(\$ 96, \$ 24),(\$ 480, \$ 0),(\$ 480, \$ 30)$, $(\$ 480, \$ 120),(\$ 960, \$ 0),(\$ 960, \$ 60)$, and $(\$ 960, \$ 24)$.

The second design was a $5 \times 5$, Probability by Amount design, with the smaller amount always fixed to $\$ 0$. Probabilities were the same as those used in the first design, and Amounts were $\$ 6, \$ 24, \$ 30, \$ 60$, or $\$ 120$.

The third design included six gambles with a probability of 0.5 to win one amount, otherwise the other. Amount pairs were $(\$ 6, \$ 24),(\$ 24, \$ 30),(\$ 30, \$ 60),(\$ 60, \$ 96),(\$ 96, \$ 120)$, and $(\$ 480, \$ 960)$. 
The remaining three designs were identical to the first three, except that positive amounts were converted to negative amounts (losses), producing undesirable gambles with negative expected values.

\section{Procedure}

Subjects were presented with booklets containing instructions, 10 warm-up gambles, and 76 experimental gambles with all positive (or all negative) expected values. Then subjects were given a new set of instructions, 10 additional warm-ups, followed by 76 experimental gambles with all negative (or all positive) expected values. Half of the subjects were given gambles with positive outcomes first; the other half received gambles with negative outcomes first. Since an analysis of variance showed no significant effects of order, results are averaged across the two orders.

\section{Participants}

Ninety-six undergraduates from the University of California at Berkeley were given gambles with positive and negative expected values. Subjects received credit in undergraduate psychology courses for their participation. Sixty-seven undergraduates from California State University, Fullerton, who received extra credits in introductory psychology for participating, received only the first three designs (76 gambles with positive expected values). Analysis of variance detected no significant differences between the Fullerton students and Berkeley students, so results for positive gambles are pooled across groups. A few additional subjects from each group who did not follow instructions were excluded from the analyses.

\section{Results}

Exhibits 2 and 3 present mean judged prices for positive and negative two-outcome gambles, respectively. Means replicate the pattern of dominance violation found in previous research. Gambles are denoted $(Y, P ; X)$, representing a gamble with probability, $P$, of outcome $Y$, otherwise outcome $X$, where $|Y|>|X|$. In Exhibit 2, mean judgments of price are plotted against the probability of winning $Y$, with separate panels for $Y=\$ 96, \$ 480$, or $\$ 960$. Dashed curves with open circles represent gambles for which $X=\$ 0$. Solid curves with filled circles show gambles for which $X=\$ 24, \$ 120$, or $\$ 240$, in the three panels, respectively.

If judgments obeyed the dominance principle, open circles $(X=\$ 0)$ should always fall below filled circles $(X=\$ 24)$. However, when $P$ exceeds 0.8 , open circles are above filled circles in five of the six cases, indicating that mean judgments are higher for inferior gambles. When $P$ is 0.95 , open circles are above filled circles in all three cases, as predicted by configural-weight theory. If dominance were a fifty-fifty proposition, the probability that all six means would show violations is $(0.5)^{6}$, so it is unlikely that this pattern is due to noise alone. Furthermore, $55 \%, 48 \%$, and $45 \%$ of the subjects offer to pay more to play the inferior gamble, when $Y=\$ 96, \$ 480$, and $\$ 960$, respectively. Only $23 \%, 29 \%$, and $26 \%$ conformed to dominance by assigning lower prices to the inferior gamble. The others assign the same value to both gambles.

Von Winterfeldt et al. (1993) investigated dominance violations for the gambles shown in Exhibit 2, using the same stimuli and procedure. They found that when the probability of winning $Y$, the larger amount, was $0.95,35 \%, 42 \%$, and $51 \%$ of their subjects violated the dominance principle, for $Y=\$ 96$, $\$ 480$, and $\$ 960$, respectively. Although these percentages are smaller than those in the present study, they are not significantly different.

Dominance violations in the domain of losses (Exhibit 3) appear to be the reflection of those in the domain of gains. In all three panels, open circles and dashed lines cross solid circles and solid lines. When 


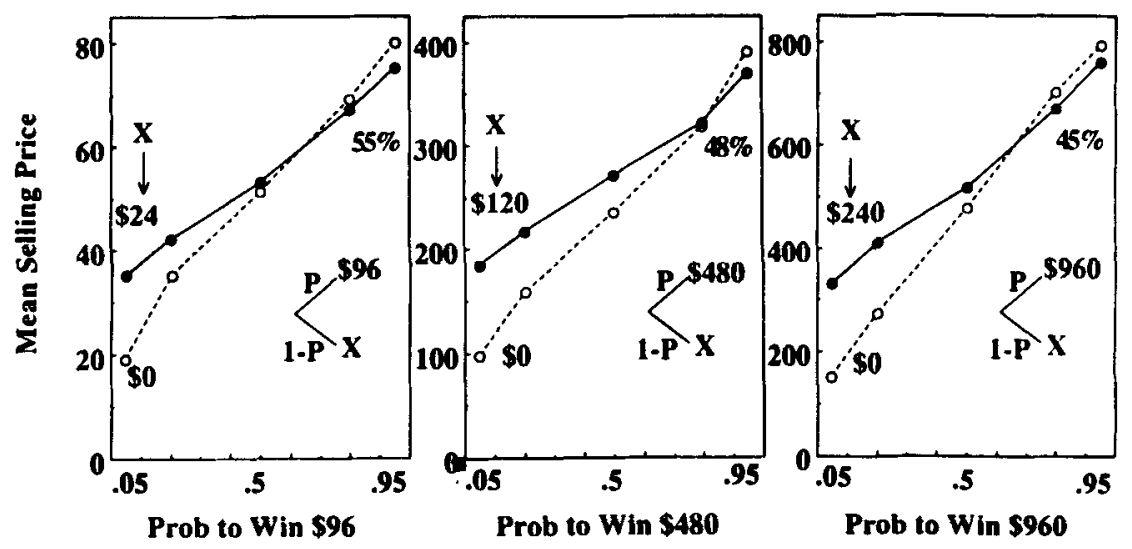

Exhibit 2. Mean judged prices to sell positive two-outcome gambles. Open and filled circles show mean judgments, plotted as a function of the probability of winning the larger outcome $(Y)$ with separate panels for $Y=\$ 96, \$ 480$, and $\$ 960$. A separate curve is drawn for each level of the lower outcome, $X$. Open circles represent judged prices of gambles for which $X=\$ 0$, and filled circles depict judgments of gambles when $X=\$ 24, \$ 120$, or $\$ 240$ in the three panels, respectively. Means are averaged over 163 subjects (Experiment 1)

the probability of losing $Y$ is $0.95,52 \%, 46 \%$, and $47 \%$ of the subjects pay more to avoid the better gamble, with a loss of $Y$, otherwise, $\$ 0$, when $Y$ is $-\$ 96,-\$ 480$, and $-\$ 960$, respectively. Far fewer subjects $(27 \%, 21 \%$, and $20 \%)$ pay more to avoid the worse gamble, with a loss of $Y$, otherwise a loss of $X$. The remaining subjects had tied responses.

Can dominance violations be explained by noise or error? To answer this question another way, we compared the rate of violations in a gamble pair for which one gamble had a zero outcome to the rate of

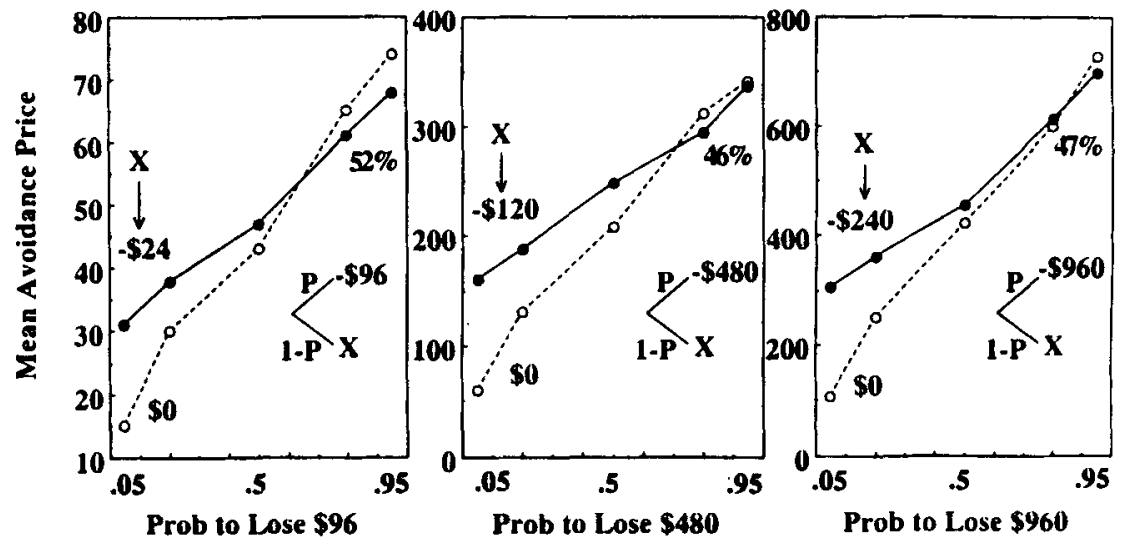

Exhibit 3. Mean prices offered to avoid playing undesirable gambles, plotted for easy comparison with Exhibit 2. Larger numbers on the ordinate represent greater (absolute) amounts offered to avoid the gamble ( $X$ and $Y$ represent amounts to lose). Means are averaged over 96 subjects (Experiment 1) 
violations in a pair for which neither gamble had a zero outcome. For example, dominance violations for the pair $(\$ 96,0.95 ; \$ 0)$ versus $(\$ 96,0.95 ; \$ 24)$ were compared to those for the pair $(\$ 96,0.95 ; \$ 6)$ versus $(\$ 96,0.95 ; \$ 24)$. Configural-weight theory predicts that there should be more violations with first pair than the second. But if dominance violations were simply noise, one would expect more violations with the second pair than with the first, because the difference between expected values for the second pair is smaller than that for the first.

Exhibits 4 and 5 present rates of dominance violations for the domains of gains and losses, respectively. Tied responses are included with nonviolations. We compared the two proportions on the off-diagonals cells of each box (e.g. 14\% versus 7\% in the upper box of Exhibit 4). These cells represent the proportion of times subjects violated dominance in one pair, but not the other. If zero is a key ingredient, the number in the lower left cell should be greater than in the upper cell. This pattern occurred in all six cases, a result that would be unlikely under the null hypothesis that both types of violations are equally likely $(p=0.016)$.

When proportions were tested individually, two of the six contrasts were significantly different (Exhibit 4, $Y=\$ 96, X^{2}(1)=4.24$, and Exhibit 5, $Y=-\$ 96, X^{2}(1)=4.84$ ). Zero is a critical component for producing dominance violations, even when pitted against a greater difference in outcomes for $X(\$ 0$ and $\$ 24$ versus $\$ 6$ and $\$ 24$ ). These results are consistent with configural-weight theory, using the parameters in Exhibit 1. The finding that all six contrasts are in the opposite direction as that predicted differences in EVs leads us to reject the hypothesis that dominance violations can be explained by noise alone.

\section{EXPERIMENT 2: TWO- AND THREE-OUTCOME GAMBLES}

Experiment 2 is designed to investigate dominance violations in both two- and three-outcome gambles. We consider two types of three-outcome gambles, those having two similar outcomes and those with three different outcomes. Since people might treat gambles with two similar outcomes as a two-outcome gamble, we manipulated the similarity of outcomes for the two higher consequences.

\section{Method}

Experiment 2 had two conditions with different subjects serving in each. In the Baseline condition $(N=$ 57), undergraduates at the University of California at Berkeley received course credit for their participation. In the Incentives condition $(N=44)$, Berkeley undergraduates received $\$ 5.00$ for their partici-

Exhibit 4. Dominance violations for two-outcome gambles with positive expected values

\begin{tabular}{lccc}
\hline & \multicolumn{2}{c}{$(\$ 96,0.95 ; \$ 24)$} & versus $(\$ 96,0.95 ; \$ 0)$ \\
& & Violations & Nonviolations \\
$(\$ 96,0.95 ; \$ 24)$ & Violations & $41 \%$ & $7 \%$ \\
versus $(\$ 96,0.95 ; \$ 6)$ & Nonviolations & $14 \%$ & $38 \%$ \\
& & $(\$ 480,0.95 ; \$ 120)$ versus $(\$ 480), 0.95 ; \$ 0)$ \\
$(\$ 480,0.95 ; \$ 120)$ & Violations & Nonviolations \\
versus $(\$ 480,0.95 ; \$ 30)$ & Violations & $35 \%$ & $9 \%$ \\
& & $13 \%$ & $43 \%$ \\
& & $(\$ 960,0.05 ; \$ 240)$ versus $(\$ 960,0.95 ; \$ 0)$ \\
$(\$ 960,0.95 ; \$ 240)$ & Violations & $29 \%$ & Nonviolations \\
versus $(\$ 960,0.95 ; \$ 60)$ & Nonviolations & $16 \%$ & $11 \%$ \\
\hline
\end{tabular}

Note: Ties are included with nonviolations. 
Exhibit 5. Dominance violations for two-outcome gambles with negative expected values

\begin{tabular}{|c|c|c|c|}
\hline \multirow{4}{*}{$\begin{array}{l}(-\$ 96,0.95 ;-\$ 24) \\
\text { versus }(-\$ 96,0.95 ;-\$ 6)\end{array}$} & \multirow{4}{*}{$\begin{array}{c}\text { Violations } \\
\text { Nonviolations }\end{array}$} & \multicolumn{2}{|c|}{$(-\$ 96,0.95 ;-\$ 24)$ versus $(-\$ 96,0.95 ; \$ 0)$} \\
\hline & & Violations & Nonviolations \\
\hline & & $33 \%$ & $7 \%$ \\
\hline & & $19 \%$ & $41 \%$ \\
\hline \multirow{4}{*}{$\begin{array}{l}(-\$ 480,0.95 ;-\$ 120) \\
\text { versus }(-\$ 480,0.95 ;-\$ 30)\end{array}$} & \multirow{4}{*}{$\begin{array}{c}\text { Violations } \\
\text { Nonviolations }\end{array}$} & \multicolumn{2}{|c|}{$(-\$ 480,0.95 ;-\$ 120)$ versus $(-\$ 480), 0.95 ; \$ 0)$} \\
\hline & & Violations & Nonviolations \\
\hline & & $23 \%$ & $15 \%$ \\
\hline & & $23 \%$ & $39 \%$ \\
\hline & & $(-\$ 960,0.95$ & $(-\$ 960,0.95 ; \$ 0)$ \\
\hline & & Violations & Nonviolations \\
\hline$(-\$ 960,0.95 ;-\$ 240)$ & Violations & $34 \%$ & $8 \%$ \\
\hline versus $(-\$ 960,0.95 ;-\$ 60)$ & Nonviolations & $13 \%$ & $45 \%$ \\
\hline
\end{tabular}

Note: Ties are included with nonviolations.

pation, and these subjects were also informed they would play one of two gambles at the end of the experiment. Two gambles would be selected, and the one to which subjects assigned a higher price was the one they would be allowed to play. Payoffs given to the subjects were $3 \%$ of nominal outcomes.

\section{Stimuli and design}

Subjects were presented with 60 two-outcome gambles and 96 three-outcome gambles. The 96 threeoutcome gambles, denoted $(Y, P ; Z, Q ; X)$, were constructed from a $(4 \times 2 \times 4 \times 3)$ design of $Y$ by $P$ by $Z$ by $X$. Values of $Y$ were $\$ 12, \$ 24, \$ 48$, and $\$ 96$, and values of $P$, the probability of $Y$, covaried with $Q$, the probability of $Z$, in the following $(P, Q)$ pairs: $(0.475,0.475)$ and $(0.85,0.10)$. Values of $Z$ were $-\$ 90$, $\$ 10, \$ 10$, and $\$ 90$, and those of $X$ were $\$ 0, \$ 6$, and $\$ 20$.

Sixteen additional three-outcome gambles were constructed from two designs of $P$ by $Q$ by $X$, where $Y$ and $Z$ were fixed to $\$ 96$ and $\$ 90$, respectively. In one design, values of $P$ were 0.475 and 0.65 , and values of $Q$ were 0.1 and 0.25 . In the other design, values of $P$ and $Q$ were reversed. In both designs, values of $X$ were $\$ 0$ and $\$ 20$. Two other gambles were included $(\$ 96,0.1 ; \$ 90,0.85 ; \$ 0)$ and $\$ 96,0.1 ; \$ 90,0.85 ; \$ 20$ ).

Two-outcome gambles, denoted $(Y, P ; X)$, were constructed from a $(4 \times 5 \times 3)$ Y by $\mathrm{P}$ by $X$ factorial design. Values of $Y$ were $\$ 12, \$ 24, \$ 48$, and $\$ 96$, and values of $P$, the probability of winning $Y$, were 0.05 , $0.15,0.475,0.85$, and 0.95 . Values of $X$ were $\$ 0, \$ 6$, and $\$ 20$.

\section{Procedure}

Subjects received instructions, 10 warm-up gambles, and 156 randomly-ordered test gambles, similar to Experiment 1. After each subject completed the judgments, two gambles were selected. Although subjects were led to believe that the gambles were selected randomly, the same two gambles were chosen for all subjects. These gambles were $(\$ 96,0.475 ; \$ 90,0.475 ; \$ 0)$ and $(\$ 96,0.475 ; \$ 90,0.475 ; \$ 20)$, two gambles predicted to produce dominance violations. Judged selling prices for these gambles were compared, and subjects were asked three questions: (1) Which of these two gambles would you prefer to play? (2) If given the opportunity, would you like to change either of your selling prices? and (3) If so, what would the new selling price(s) be? 


\section{Results}

Judged prices for two-outcome gambles resembled those found in Experiment 1. Analysis of variance for judgments of two-outcome gambles showed no significant differences between Experiments 1 and 2. Mean judgments violate the dominance principle in the same fashion as reported in Experiment 1.

Three-outcome gambles with two similar outcomes

Exhibit 6 presents mean judged prices for three-outcome gambles in the Baseline condition when two of the outcomes are large and similar $(\$ 96$ and $\$ 90)$. Judged prices are plotted against $P$, the probability of winning $\$ 96$, with separate panels for different levels of $Q$, the probability of winning $\$ 90$. Open and filled circles show prices when the third outcome, $X$, is $\$ 0$ or $\$ 20$, respectively. When open circles are above filled circles, mean judgments violate the dominance principle. There are six violations of dominance in the 10 comparisons of means in Exhibit 6.

Exhibit 6 shows that when the probabilities of the two higher outcomes, $\$ 96$ and $\$ 90$, sum to 0.90 or greater, dominance is violated. This sum of 0.90 or more for the pair of circles (open and solid) on the right side of each panel. For these pairs, $51 \%, 39 \%, 49 \%$, and $49 \%$ of the subjects violate the dominance principle when $Q$ is $0.1,0.25,0.475$, and 0.65 , respectively. The percentage of subjects who obey the principle is only $24 \%, 26 \%, 23 \%$, and $14 \%$, respectively, and the rest are ties. Apparently, the particular combination of $P$ and $Q$ is not crucial; what seems to matter is that the combined probability of the two high outcomes is 0.90 or larger (hence, the probability of the lowest outcome is 0.1 or less).

Exhibit 7 shows individual rates of dominance violations for all 10 gamble pairs in Exhibit 6 . The percentage of subjects who assign higher prices to inferior gambles (i.e. Violations) is greater than the percentage who assign lower prices (Nonviolations) for all 10 pairs. The rate of ties (shown in the last column) is somewhat higher than that found with two-outcome gambles in Experiment 1 and in previous research (Mellers et al., 1992b; Birnbaum et al., 1992; Birnbaum and Sutton, 1992).

Results from the Incentives condition show that even when people are financially motivated, they violate the dominance principle. The same pattern of violations appears in the means for two of the four panels in Exhibit 6 when $Q \geqslant 0.475$ and $P+Q \geqslant 0.9$. There are no significant main effects or interactions involving financial incentives. Exhibit 8 presents the individual rates of dominance violations for the 10 gamble pairs, as in Exhibit 7. A comparison of Exhibits 7 and 8 shows that percentages of violations are
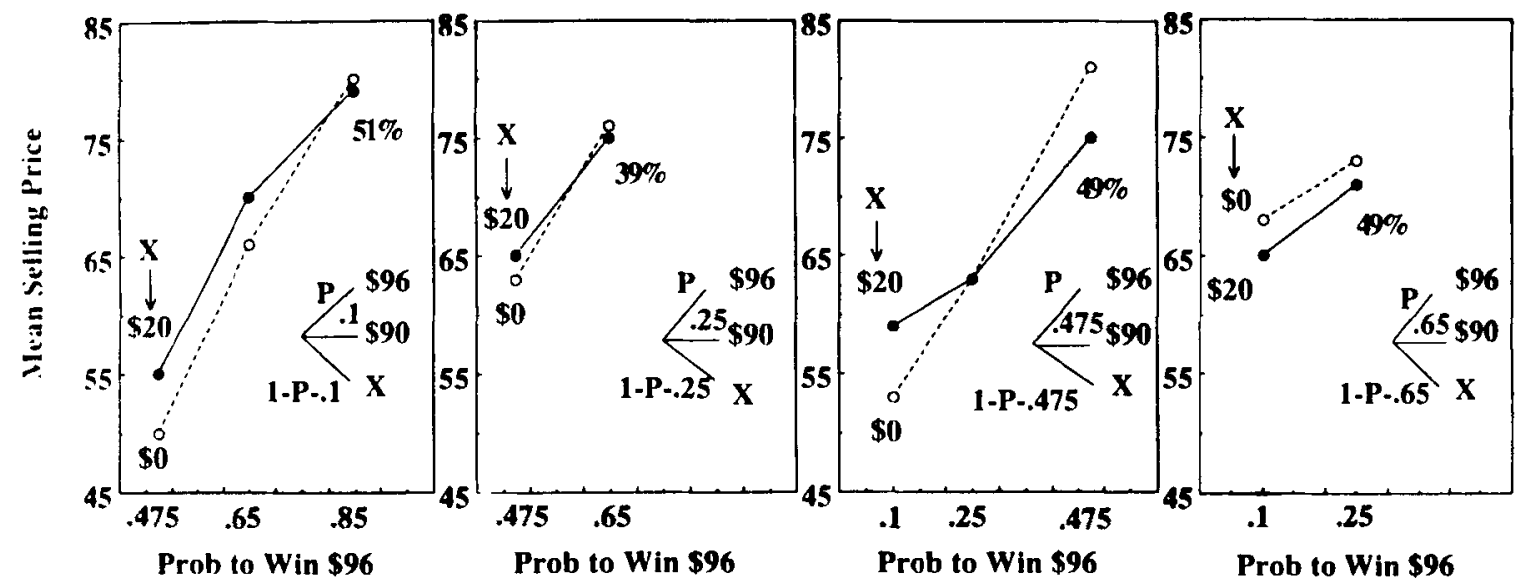

Exhibit 6. Mean judged prices for three-outcome gambles in the Baseline condition of Experiment $2(N=57)$ with two larger outcomes $(\$ 96$ and $\$ 90$ ) and variable probabilities. Open and filled circles show mean judgments for $X=$ $\$ 0$ and $\$ 20$, respectively, plotted as a function of probability to win $\$ 96(\mathrm{P})$, with a separate panel for each probability to win $\$ 90(Q=0.1,0.25,0.475$, and 0.65$)$ 
Exhibit 7. Dominance violations for three-outcome gambles in the Baseline condition $(\$ 96, p ; \$ 90, q ; \$ 0)$ versus $(\$ 96, p ; \$ 90, q ; \$ 20)$

\begin{tabular}{lllccc}
\hline \multicolumn{1}{c}{$p$} & $Q$ & $1-p-q$ & Violations (\%) & Nonviolations (\%) & Ties (\%) \\
\hline 0.475 & 0.1 & 0.425 & 28 & 12 & 60 \\
0.65 & 0.1 & 0.25 & 30 & 17 & 53 \\
0.85 & 0.1 & 0.05 & 51 & 24 & 25 \\
0.475 & 0.25 & 0.275 & 33 & 18 & 49 \\
0.65 & 0.25 & 0.10 & 39 & 26 & 35 \\
0.1 & 0.475 & 0.425 & 26 & 14 & 60 \\
0.25 & 0.475 & 0.275 & 42 & 21 & 37 \\
0.475 & 0.475 & 0.05 & 49 & 23 & 28 \\
0.1 & 0.65 & 0.25 & 30 & 14 & 49 \\
0.25 & 0.65 & 0.10 & 49 & & 37 \\
\hline
\end{tabular}

reduced for 7 out of the 10 gamble pairs, and ties are less frequent with financial incentives than without. In summary, cash incentives appear to have reduced the proportion of ties and increased conformity to dominance, nevertheless, systematic dominance violations persist, and the means are not significantly changed by incentives.

Judged prices for three-outcome gambles in Exhibit 6 were predicted from configural-weight theory, using the simplified decision weights shown in Exhibit 1 and utilities that were set to monetary amounts, as in Birnbaum et al. (1992). This procedure estimates no parameters from the new data; instead, it uses simplified approximation of the parameters from the seller's condition of Birnbaum et al. to calculate predictions. Predictions, shown in Exhibit 9, resemble the data in the Baseline and Incentives conditions. Even with nonoptimal parameters taken from the previous research, configural-weight theory accounts for the dominance violations and describes the major trends in the data.

\section{Three-outcome gambles with different outcomes}

Since $\$ 90$ and $\$ 96$ are so similar, subjects might treat those two outcomes as essentially one, and the gambles in Exhibit 6 would be perceived as having two outcomes, rather than three. Exhibit 10 presents mean judged prices for gambles when one outcome is $\$ 96$, and the second outcome ranges from $\$ 90$ to $-\$ 90$. Judged prices are shown as a function of $P$, the probability of winning $\$ 96$, with a separate curve for each value of $X$, the third outcome. Open and filled circles represent gambles when $X$ is either $\$ 0$ or $\$ 20$, respectively. Separate panels are shown for each value of $Z$ and range from $\$ 90$ to $-\$ 90$.

When $P$, the probability of $\$ 96$, is 0.85 , mean judgments violate the dominance principle. Open and

Exhibit 8. Dominance violations for three-outcome gambles in the Incentives condition $(\$ 96, p ; \$ 90, q ; \$ 0)$ versus $(\$ 96, p ; \$ 90, q ; \$ 20)$

\begin{tabular}{lllccc}
\hline \multicolumn{1}{c}{$p$} & $Q$ & $1-p-q$ & Violations (\%) & Nonviolations (\%) & Ties (\%) \\
\hline 0.475 & 0.1 & 0.425 & 32 & 59 & 9 \\
0.65 & 0.1 & 0.25 & 25 & 55 & 30 \\
0.85 & 0.1 & 0.05 & 34 & 45 & 21 \\
0.475 & 0.25 & 0.275 & 48 & 48 & 4 \\
0.65 & 0.25 & 0.10 & 34 & 45 & 21 \\
0.1 & 0.475 & 0.425 & 18 & 51 & 11 \\
0.25 & 0.475 & 0.275 & 36 & 30 & 7 \\
0.475 & 0.475 & 0.05 & 45 & 41 & 25 \\
0.1 & 0.65 & 0.25 & 45 & 45 & 14 \\
0.25 & 0.65 & 0.10 & 41 & & 14 \\
\hline
\end{tabular}




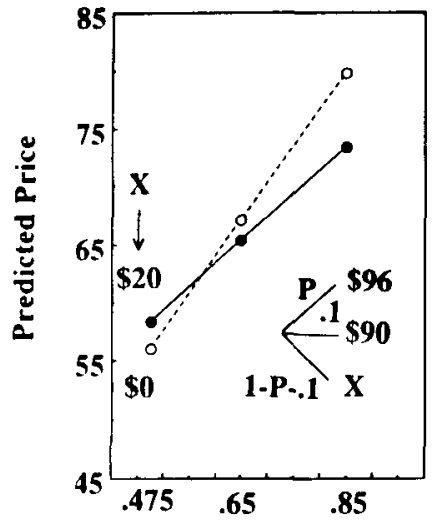

Prob to Win $\$ 96$

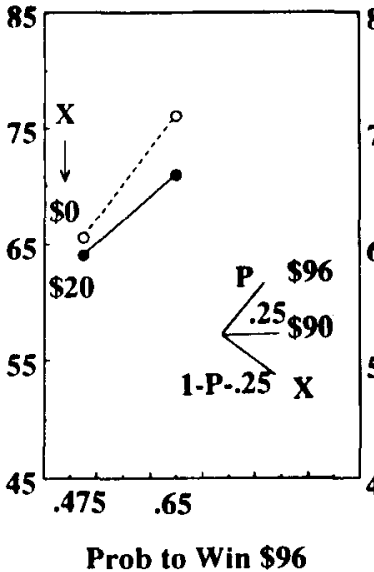

Prob to Win $\$ 96$
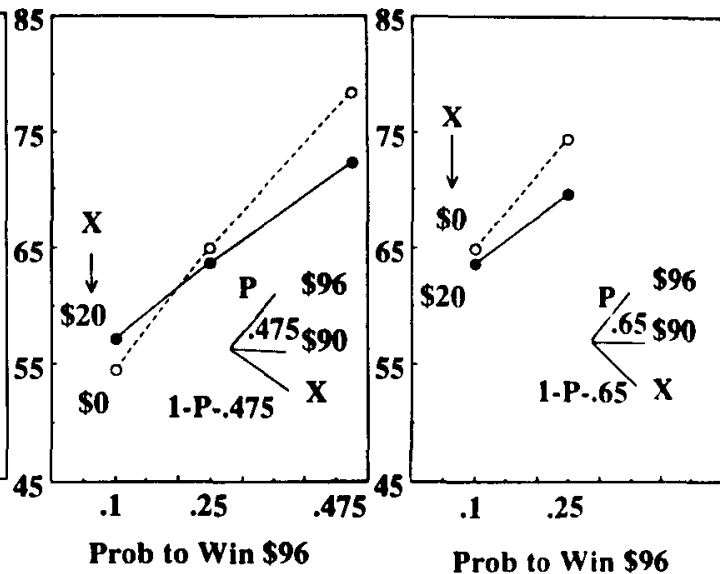

Exhibit 9. Predictions of configural-weight theory for judged prices shown in Exhibit 6, using approximate decision weights from Birnbaum et al. (1992), from Exhibit 1

solid circles on the far right of each panel represent gambles for which $P$ is 0.85 . Although these dominance violations are small, they are systematic. Open circles are above solid circles for all values of $Z$ except $-\$ 90$. For these pairs of gambles, $51 \%, 54 \%, 53 \%$, and $46 \%$ of the subjects violate dominance when $Z$ is $\$ 90, \$ 10,-\$ 10$, and $-\$ 90$, respectively. The percentage of subjects who obey the principle is only $24 \%, 16 \%, 14 \%$, and $9 \%$, respectively, and the rest are ties. Exhibit 11 presents individual rates of dominance violations for the eight pairs of gambles in Exhibit 10. The percentage of violations exceeds the percentage of nonviolations for all pairs. Thus, subjects violate dominance in three-outcome gambles, even when the three outcomes differ.

When subjects are given financial incentives, similar patterns emerge in the mean judgments. Exhibit 12 presents the rate of dominance violations for the eight gamble pairs from the Incentives condition. A comparison of Exhibits 11 and 12 shows that the percentage of subjects making violations is reduced for six out of eight gamble pairs. However, an analysis of variance on the baseline and financial conditions
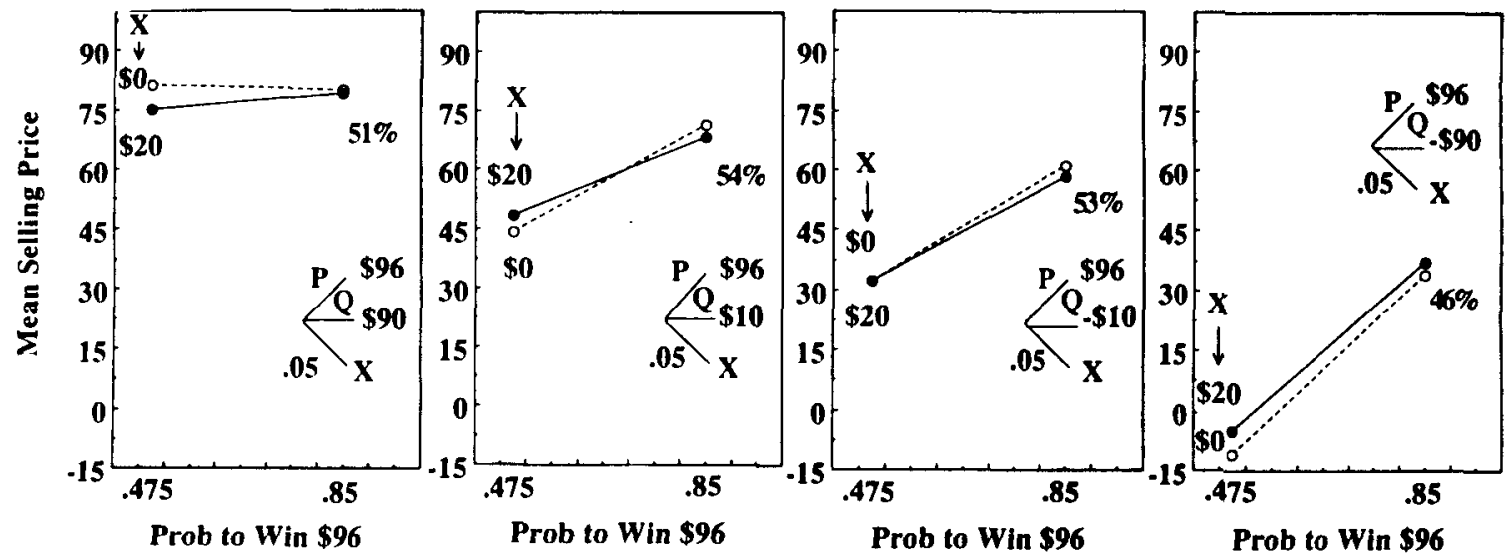

Exhibit 10. Mean judged prices for three-outcome gambles in the Baseline condition of Experiment 2 with one outcome of $\$ 96$, and different values of $Z$. Judged prices are plotted against the probability of winning $\$ 96$, with a separate panel for $Z=\$ 90, \$ 10,-\$ 10$, and $-\$ 90$. Open circles show judged prices for gambles when $X=\$ 0$, and filled circles are prices when $X=\$ 20$ 
Exhibit 11. Dominance violations for three-outcome gambles in the Baseline condition $(\$ 96, p ; \$ X, q: \$ 0)$ versus $(\$ 96, p ; \$ \mathrm{X}, q ; \$ 20)$

\begin{tabular}{lllccc}
\hline$p$ & $Q$ & $X$ & Violations (\%) & Nonviolations (\%/) & $\begin{array}{c}\text { Ties } \\
(\%)\end{array}$ \\
\hline 0.475 & 0.475 & 90 & 49 & 23 & 28 \\
0.85 & 0.10 & 90 & 51 & 24 & 25 \\
0.475 & 0.475 & 10 & 35 & 9 & 56 \\
0.85 & 0.10 & 10 & 54 & 16 & 30 \\
0.475 & 0.475 & -10 & 44 & 12 & 44 \\
0.85 & 0.10 & -10 & 53 & 14 & 33 \\
0.475 & 0.475 & -90 & 35 & 9 & 51 \\
0.85 & 0.10 & -90 & 46 & 95 \\
\hline
\end{tabular}

shows that the mean judgments are not significantly different. Nonetheless, the percentage of tied responses is smaller, and the percentage of responses that conform to dominance is larger.

Predictions from configural-weight theory are shown in Exhibit 13. Configural-weight theory predicts dominance violations in the first two panels (when $Z=\$ 90$ and $Z=\$ 10$ ), but not the latter two (when $Z=$ $-\$ 10$ and $Z=-\$ 90)$. A comparison of the predictions with the data in Exhibit 10 shows that three of the four predicted violations and one unpredicted violation occur. Dominance violations occur in the mean when $Z$ is $-\$ 10$, which was not predicted. However, results from the other panels are reasonably well predicted by the theory.

In summary, a comparison of Exhibits 6 and 9 shows that there are eight predicted violations out of 10 comparisons; six of these eight violations occur in the data, and there are no unpredicted violations. A comparison of Exhibits 10 and 13 shows that there are four predicted violations of dominance out of eight comparisons. Three of the predicted violations also occur in the data, and one additional violation occurs that was not predicted. In sum, configural-weight theory predicted 12 dominance violations out of 18 comparisons of three-outcome gambles. The empirical means confirm nine of the 12 predicted violations and one that was unpredicted.

\section{Post-experimental interviews}

When confronted with their decisions, subjects who violate normative principles sometimes try to defend or justify their responses (Tversky, 1969; Lichtenstein and Slovic, 1971; Slovic and Tversky, 1974). However, none of our subjects defended their violations of dominance. When asked which of the two gambles they preferred to play, subjects always preferred the dominant gamble. When asked why they had made their responses, those who violated dominance said that they had either made a 'mistake' or had been imprecise. One subject said that if he had seen the gambles together, he would not have made

Exhibit 12. Dominance violations for three-outcome gambles in the Incentives condition $(\$ 96, p ; \$ \mathrm{X}, q ; \$ 0)$ versus $(\$ 96, p ; \$ \mathrm{X}, q \$ 20$ )

\begin{tabular}{lllccr}
\hline$p$ & $Q$ & $X$ & Violations (\%) & Nonviolations (\%/) & $\begin{array}{c}\text { Ties } \\
(\% / 1)\end{array}$ \\
\hline 0.475 & 0.475 & 90 & 45 & 30 & 25 \\
0.85 & 0.10 & 90 & 34 & 45 & 21 \\
0.475 & 0.475 & 10 & 32 & 50 & 18 \\
0.85 & 0.10 & 10 & 55 & 36 & 9 \\
0.475 & 0.475 & -10 & 41 & 41 & 18 \\
0.85 & 0.10 & -10 & 50 & 36 & 14 \\
0.475 & 0.475 & -90 & 36 & 41 & 23 \\
0.85 & 0.10 & -90 & 36 & 55 & 9 \\
\hline
\end{tabular}




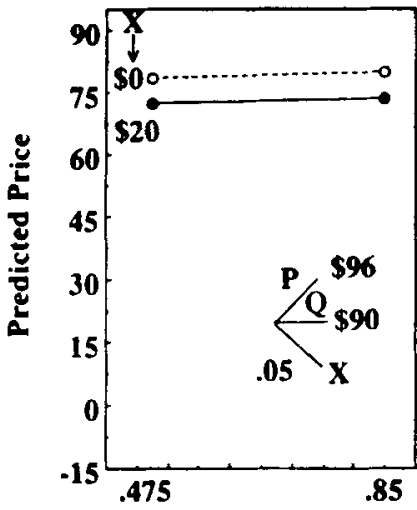

Prob to Win $\$ 96$

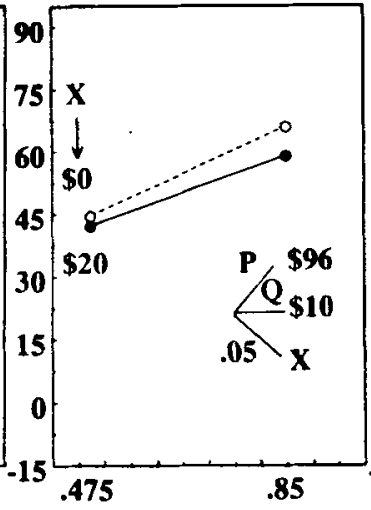

Prob to Win $\$ 96$

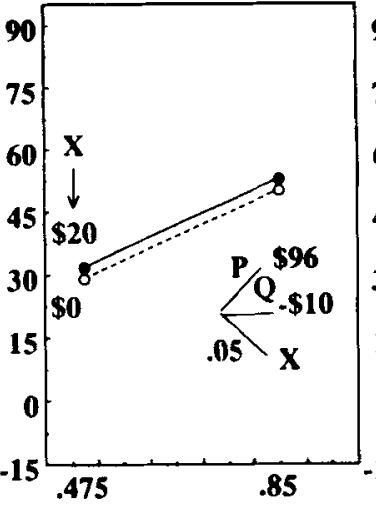

Prob To Win $\$ 96$

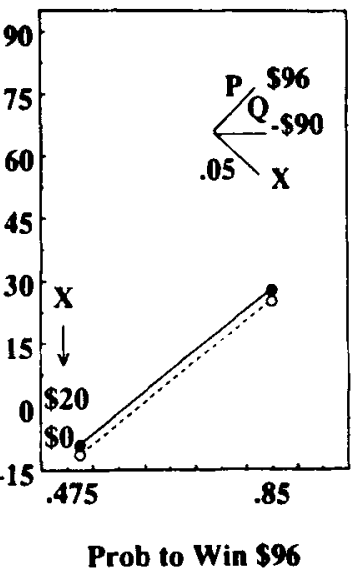

Exhibit 13. Predictions of configural-weight theory for gambles shown in Exhibit 10, using simplified decision weights from Birnbaum et al. (1992), shown in Exhibit 1

the mistake, but if he were given the same two gambles separately on another occasion, he was sure he would do the same as before. When asked whether they would like to change either or both of their judgments, a large majority said they would simply reverse their judged prices.

\section{DISCUSSION}

\section{Two- and three-outcome gambles}

Results from Experiment 1 and the two-outcome gambles of Experiment 2 replicate earlier work of Mellers et al. (1992b), Birnbaum et al. (1992), Birnbaum and Sutton (1992), Birnbaum (1992), and Birnbaum and Thompson (in press) by finding the predicted pattern of dominance violations with two-outcome gambles. Results from Experiment 2 extend the work by showing that dominance violations are not unique to two-outcome gambles; they also occur with three-outcome gambles (Exhibits 6 and 10). Furthermore, subjects who are paid to participate and given the opportunity to play one of the gambles for a cash prize still violate the dominance principle.

The present data show that there are at least two ways to create dominance violations with threeoutcome gambles. One way is to use gambles with two similar outcomes, such as $\$ 96$ and $\$ 90$. When the combined probability of their occurrence is 0.90 or greater, people tend to assign a higher price to the inferior gamble (with a third outcome of $\$ 0$ ) than the superior gamble (with a third outcome of $\$ 20$ ). The other way is to use gambles with a large probability of a relatively large outcome, such as an $85 \%$ chance of $\$ 96$. For a wide range of values of the second outcome (i.e. $\$ 90$ to $-\$ 10$ ), subjects assign a higher price to gambles with a third outcome of zero than with a third outcome of $\$ 20$.

\section{Configural-weight theory}

The fact that subjects violate dominance with three-outcome gambles rules out the possibility that violations are the result of a simplifying strategy applied only to two-outcome gambles. Dominance violations for gambles with both two and three outcomes are well described by configural-weight theory. According to configural-weight theory, decision weights depends on the judge's point of view (e.g. buyer's versus seller's), the rank of the outcome, and the value of the outcome (zero versus nonzero). Zero-valued outcomes have less weight relative to nonzero outcomes, especially for small probabilities 
(Exhibit 1). Less weight for the zero-valued outcome implies greater relative weight for the nonzero outcome, since relative weight must sum to 1 . This shift in decision weight predicts the patterns of dominance violations found in both two- and three-outcome gambles.

Configural-weight theory also explains the fact that buyers prices are often lower than sellers prices (Coombs et al., 1967; Knetsch and Sinden, 1984). Birnbaum and Stegner (1979) and Birnbaum et al. (1992) have found that the rank order of gambles, as well as the value of gambles, changes across different points of view. Consider two gambles, Gamble A with a $50 \%$ chance of $\$ 78$, otherwise $\$ 72$, and Gamble B, with a $95 \%$ chance of winning $\$ 96$, otherwise $\$ 0$. For both of these gambles, buyers prices are lower than sellers prices. In addition, buyers will pay more to play Gamble A than Gamble B, and sellers want more to sell Gamble B than Gamble A. These differences in rank order are predicted by configural-weight theory because buyers tend to assign greater weights to lower-valued outcomes, whereas sellers assign greater weights to higher-valued outcomes. The rank-dependent aspect of configural-weight theory has been incorporated into theories of impression formation, moral judgment, and evaluative preferences (Birnbaum, 1973, 1974) as well as theories of choice (Quiggin, 1982; Lopes, 1984; Luce and Narens, 1985; Luce, 1991; Luce and Fishburn, 1991; Tversky and Kahneman, 1992).

In conclusion, when people evaluate the worth of gambles using judged prices, they violate the most fundamental principle of decision making. This result might lead some researchers to argue that choices, and not judgments, should be used to measure preference. Although Birnbaum and Sutton (1992) found that the dominance principle was satisfied using direct choice with a transparent dominance relationship, Birnbaum and Thompson (in press) found that most subjects prefer $(\$ 0,0.05 ; \$ 96)$ to $\$ 70$, but they choose $\$ 70$ over $(\$ 24,0.05 ; \$ 96)$. Therefore indirect choices can also violate dominance. Furthermore, choices can violate weak stochastic transitivity (Tversky, 1969), whereas judgments automatically satisfy this axiom. Both choices and judgments violate fundamental principles of decision making and provide serious challenges to principles of rationality.

\section{ACKNOWLEDGEMENTS}

The authors thank R. Duncan Luce and Detlof von Winterfeldt for stimulating discussions and Katty Ho and Laura A. Thompson for assistance in data collection. This research was supported by National Science Foundation grants (SES-90-23160) to the first author and (SES-8921880) to the third author. Requests for reprints should be addressed to Barbara A. Mellers, Department of Psychology, University of California, Berkeley, CA 94720 . E-mail correspondence can be sent to mellers@violet.berkeley.edu or mbirnbaum@fullerton.edu.

\section{REFERENCES}

Arkes, H. R. and Hammond, K. R. (Eds) Judgment and Decision Making: An Interdisciplinary Reader, Cambridge: Cambridge University Press, 1986.

Bell, D. E., Raiffa, H. and Tversky, A. (Eds) Decision making: Descriptive, Normative, and Prescriptive Interactions, Cambridge: Cambridge University Press, 1988.

Birnbaum, M. H. 'Morality judgment; Test of an averaging model with differential weights', Journal of Experimental Psychology, 99, (1973), 395-99.

Birnbaum, M. H. 'The nonadditivity of personality impressions', Journal of Experimental Psychology, 102, (1974), 543-61 (monograph).

Birnbaum, M. H. 'Violations of monotonicity and contextual effects in choice-based certainty equivalents', Psychological Science, 3 (1992), 310-14.

Birnbaum, M. H., Coffey, G., Mellers, B. A. and Weiss, R. 'Utility measurement: Configural-weight theory and the 
judge's point of view', Journal of Experimental Psychology: Human Perception and Performance, 18, (1992), $331-46$.

Birnbaum, M. H. and Stegner, S. E. 'Source credibility in social judgment: Bias, expertise, and the judge's point of view', Journal of Personality and Social Psychology, 37, (1979), 48-74.

Birnbaum, M. H. and Sutton, S. E. 'Scale convergence and utility measurement', Organizational Behavior and Human Decision Processes, 52 (1992), 183-215.

Birnbaum, M. H. and Thompson, L. 'Violations of monotonicity in choices between gambles and certain cash', American Journal of Psychology, XX, in press.

Coombs, C. H., Bezenbinder, T. G. and Goode, F. M. 'Testing expectation theories of decision making without measuring utility or subjective probability', Journal of Mathematical Psychology, 4 (1967), 72-103.

Knetsch, J. L. and Sinden, J. A. 'Willingness to pay and compensation demanded: Experimental evidence of an unexpected disparity in measures of value', Quarterly Journal of Economics, 99 (1984), 507-21.

Lichtenstein, S. and Slovic, P. 'Reversals of preferences between bids and choices in gambling decisions', Journal of Experimental Psychology, 89 (1971), 46-55.

Lopes, L. 'Risk and distributional inequality', Journal of Experimental Psychology: Human Perception and Performance, 10 (1984), 465-85.

Luce, R. D. 'Risk- and sign-dependent linear utility models for binary gambles', Journal of Economic Theory, 53 (1991), 75-100.

Luce, R. D. and Fishburn, P. C. 'Rank- and sign-dependent linear utility models for finite first-order gambles', Journal of Risk and Uncertainty, 4 (1991), 29-59.

Luce, R. D. and Narens, L. 'Classification of concatenation measurement structures according to scale type', Journal of Mathematical Psychology, 29 (1985), 1-72.

Mellers, B. A., Weiss, R. and Birnbaum, M. H. 'Violations of dominance in pricing judgments', Journal of Risk and Uncertainty, 5 (1992a), 73-90.

Mellers, B. A., Chang, S., Birnbaum, M. H. and Ordóñez, L. 'Preferences, prices, and ratings in risky decision making', Journal of Experimental Psychology: Human Perception and Performance, 18 (1992b), 347-61.

Quiggin, J. 'A theory of anticipated utility', Journal of Economic Behavior and Organization, 3 (1982), 32443.

Savage, L. L. Foundations of Statistics, New York: Dover, 1954.

Slovic, P. and Tversky, A. 'Who accepts Savage's axioms?' Behavioral Science, 19 (1974), 368-73.

Tversky, A. 'Intransitivities of preferences', Psychological Review, 34 (1969), 273-86.

Tversky, A. and Kahneman, D. 'Advances in prospect theory: Cumulative representation of uncertainty', Journal of Risk and Uncertainty, 5 (1992), 297-323.

von Winterfeldt, D., Chung, N., Luce, R. D. and Cho, Y. 'Tests of consequence monotonicity in decision making under uncertainty', Working paper, 1993.

Wakker, P. 'Separating marginal utility and probabilistic risk aversion', Theory and Decision, 36 (1994), 1-44.

Yaari, M. E. 'The dual theory of choice under risk', Econometrica, 55 (1985), 95-115.

Authors' biographies:

Barbara Mellers is Full Professor in the Psychology Department at the University of California at Berkeley.

Patricia Berretty was an undergraduate at the University of California at Berkeley when this research was done. She is now a graduate student at the University of California, Santa Barbara.

Michael Birnbaum is Full Professor at the California State University at Fullerton.

Authors' addresses:

Barbara A. Mellers, Department of Psychology, University of California, Berkeley, CA 94720, USA.

Patricia M. Berretty, Department of Psychology, University of California, Santa Barbara, CA, USA.

Michael H. Birnbaum, Department of Psychology, California State University, Fullerton, CA 92634, USA. 